\title{
A Framework for a Decision Support System in a Hierarchical Extended Enterprise Decision Context
}

\author{
Andrés Boza, Angel Ortiz, Eduardo Vicens, and Raul Poler \\ Research Centre on Production Management and Engineering (CIGIP), \\ Universidad Politécnica de Valencia, \\ Camino de Vera s/n. Ed 8G - Esc. 4 - Nivel 1, \\ 46022 - Valencia, Spain \\ \{aboza, aortiz, evicens, rpoler\} @cigip.upv.es
}

\begin{abstract}
Decision Support System (DSS) tools provide useful information to decision makers. In an Extended Enterprise, a new goal, changes in the current objectives or small changes in the extended enterprise configuration produce a necessary adjustment in its decision system. A DSS in this context must be flexible and agile to make suitable an easy and quickly adaptation to this new context. This paper proposes to extend the Hierarchical Production Planning (HPP) structure to an Extended Enterprise decision making context. In this way, a framework for DSS in Extended Enterprise context is defined using components of HPP. Interoperability details have been reviewed to identify the impact in this framework. The proposed framework allows overcoming some interoperability barriers, identifying and organizing components for a DSS in Extended Enterprise context, and working in the definition of an architecture to be used in the design process of a flexible DSS in Extended Enterprise context which can reuse components for futures Extended Enterprise configurations.
\end{abstract}

Keywords: Decision Support System, Extended Enterprise, Interoperability, Hierarchical Production Planning.

\section{Introduction}

The decision system allows to reach the stated objectives in the organizations. In order to provide the necessary information for decision-making, the information system becomes a key element within this decision making process. A Decision Support System (DSS), as a computer technology solution that can be used to support complex decision-making [1], relates to decision and information systems.

The increasing collaboration among enterprises during the entire product life cycle and constant changes in inter and intra organizational environment is a trend in the global market [2]. This is generating news inter-enterprise relationships like Extended Enterprise or Virtual Enterprise.

An Extended Enterprise is defined as the formation of closer coordination in the design, development, costing and the coordination of the respective manufacturing schedules of cooperating independent manufacturing enterprises and related suppliers [3]. Relationships between the participating enterprises are for a long term integration 
and more stable than other configurations like Virtual Enterprise [4]. In this decision context, the decision process is more complex than inside a single organization and a DSS for an extended enterprise must consider these inter-dependencies [5]. Environment changes where organizations are becoming more complex yet more agile and flexible, and global regulatory, and competitive factors rapidly change, affecting the design and use of these tools [1].

On the other hand, the legacy software systems play a key role in any interorganizational DSS. These legacy systems, located in the participating enterprises, are the main data source for the DSS. In this sense, ERP users highlight the importance of decision support and trans-organizational objectives in ERP planning.

This paper proposes a framework for a Decision Support System in a Hierarchical Extended Enterprise Decision Making (HEEDM) context. We make a hierarchical approach to this decision context, where the coordination between decision activities in strategic, tactical and operative levels is made according to a hierarchical structure. This means that each one will pursue its own goals, but always considering those of superior levels, on which they depend, and those of inferior levels, at which they restrict. We propose to extend the Hierarchical Production Planning structure to a HEEDM context. In this way, a framework is defined using components of Hierarchical Production Planning.

\section{Hierarchical Production Planning}

In the Hierarchical Production Planning (HPP) systems, the decisions are split in subproblems. Each sub-problem is referred to a decision-making level in the organizational structure and an optimization model is constructed for solving each sub-problem. To ensure effective decision-making, a linkage must exist between these models at each hierarchical level and an adequate aggregation/disaggregation process of information between levels. The decisions that are taken in an upper level impose restrictions on lower decision levels. In response, the detailed decisions provide the necessary feedback to evaluate the quality of the decision. Each hierarchical level has its own characteristics, including length of the decision horizon, level of detail of the required information and forecast, scope of the decision, and type of manager in charge of executing the decision [6][7][8][9][10][11][12][13]. The utility to use this hierarchic approach in interenterprise systems appears in works as [13][14][15][16][17]. Some modeling frameworks provide a structured approach to model the decision system, e.g. the GRAI model divide the decision system into level of decision-making and can be used in to provide a methodology for the design of the hierarchical production planning systems [13].

The Information System is a key tool for the HPP systems and appears since the first works in HPP, by example, [6] include technical characteristics about procedures, functions, inputs and outputs in the computer. Others works in this area have also included information about the Information System [7][18][19][20][21][22][23][13][24][25].

\section{Logical Constructs for Information and Decision Systems}

According to the Principle of Graduated Flexibility: “A model's mathematical structure and detailed data should be represented and implemented in such a way that, over 
the lifetime of an application, the most commonly required model changes are the easiest to make, while less commonly required changes may be more difficult". The independency between model's mathematical structure and data allows defining DSS that solve these models for different data sets [26][27].

Data Modeling, Decision Modeling and Model Investigation, are logical constructs, which play a leading role both in the interaction of information systems and decision technologies, as well as in rational decision making [28]:

- Data Modeling refers to the 'structured' internal representation and external presentation of recorded facts. Broadly speaking this provides the decisionmaker with information about their decision problem.

- Decision Modeling is the development of a model, or a range of models that captures the structure as well as the decisions in respect of a given problem. These models are used to evaluate possible decisions (actions) in a given problem domain, and the probable outcomes of these actions.

- Model Analysis and Investigation refers to the instantiation of the model with data, and the evaluation of the model parameters as well as the results in order to gain confidence and insight into the model.

Following this classification, we have made a framework for a DSS in HEEDM context with the necessary components for data modeling, decision modeling and model analysis and investigation.

\section{A Framework for Decision Support Systems in Hierarchical Extended Enterprise Decision Making}

This section is structured in four parts, firstly the components of the framework are defined, secondly, the relationships between components are explained, thirdly, the main roles needed to model and operate are identified, and finally a DSS platform is introduced.

\subsection{Components}

The components are defined for data modeling, decision modeling and model analysis and investigation.

The Data Modeling components included in this framework are defined in order to obtain a structured representation of the data used in a hierarchical extended enterprise decision making context. IDEF1 [29] and E-R Diagram [30] have been used to define these components. Each decision level works with a data set. The structured representation of these data sets, by means of entities, relation and attributes, for all decision models make up the data modeling.

The Decision Modeling components are defined in order to obtain a structured representation of hierarchical decision models. The hierarchy design process [13] has been employed to define these components.

On the other hand, the Model Analysis and Investigation components are defined in order to the instantiation of the models with data. Separation of Models from problems 
statements and solvers, and separation of general structure and instantiating data [26][28] have been utilized.

\section{Data Modeling}

Decision Data Model's Entity. Represents the information maintained in organizations about physical or conceptual objects (e.g., people, places, things or ideas) and which appears in decision problems in hierarchical extended enterprise decision making (HEEDM). These elements can be described in a low or high abstraction level.

Decision Data Model's Relation. Represents an association between Decision Data Model's Entities or between instances of the same Entity.

Decision Data Model's Attribute. An attribute is a property or characteristic of a Decision Data Model's Entity or Relation. This is one element of information that is known about a particular Decision Data Model's Entity or Decision Data Model's Relation, and useful for a decision problem in HEEDM.

Decision Data Model. Subset of data model's entities, relations and attributes which are used in a single decision model. These decision data models will be instanced with data for a decision model in the Model Analysis and Investigation and will be associated with a Level Model inside the Hierarchy Level in a hierarchical extended enterprise decision.

\section{Decision Modeling}

Hierarchy. Identifier of a hierarchical ordered set of decision problems in which a complex decision problem in an extended enterprise context is divided.

Hierarchy Level. Identifier of a level in the hierarchy. Each level has associate a decision sub-problem.

Decision Model. A mathematical representation of a decision problem to find the best solution. The decision model uses the entities, relations and attributes of the problem as index, data entries, decision or bounds variables for its definition, but not their values. The decision model will be associated with a Decision Data Model.

Level Model. A Decision Data Model and a Decision Model associate to a Hierarchy Level.

Aggregation/Disaggregation Process. Each Level Model is treated at a certain degree of abstraction (e.g., products-families or machines-cells). The Aggregation/ Disaggregation Processes link information between lower and upper levels of a hierarchy.

\section{Model Analysis and Investigation}

Decision Context. Identifier of a Decision Context. A hierarchy is appropriate in a particular setting which is necessary to identify. A hierarchy could be extrapolated to others decision contexts.

Data Source. Data bases, files or spreadsheets in organization information systems where the data of a Decision Data Model are located. 
Level Resolution Process. Process to obtain Definitive Decision Data at a Level Model. This is a complex process in which others components have been identified. The following components are included in this process.

Extract, Transformation and Load (ETL) Process of Data for a Level Model. The data of the Decision Data Model associated to this Level Model are extracted, transformed and loaded from the Data Source to an Analytical Database. The Entities, Relationships and Attributes defined in the Decision Data Model are instancied from the Data Source and stored in an Analytical Database. This process uses the aggregation/disaggregation process in order to complete these Analytical Data. (e.g. an entity "worker" from the Decision Data Model is instanced in all workers from a Data Source -a company- to the Analytical Database)

Analytical Data. Data obtained from the Data Sources according to a Decision Data Model and an aggregation/disaggregation process. These Analytical Data are stored in the Analytical Database.

Solver Process. A resolution process, which is capable of understanding a Decision Model and processing a data set (Analytical Data) to produce results. The results obtained after its execution are the Decision Data.

Decision Data. Data obtained for the Solver Process after its execution with a set of Analytical Data for a Decision Model.

Definitive Decision Process. The process by which the decision-maker consider additional factors in order to make its definitive decision.

Definitive Decision Data. Data about the definitive decision taken by the decisionmaker in a Level Model. Decision Data obtained by the solver can be updated by the decision-maker in function of their judgments and choices.

\subsection{Relationships}

The relationship between the components of the framework for a DSS in HEEDM context defined above is showed in figure 1.

Concerning the relationships between components in the data modeling, these exist between entities with their attributes, between entities with other entities and the attributes that arise, and the subgroups of these components that form a valid decision data modeling for a single decision model in a hierarchical extended enterprise decision making context.

Libraries of entities, relations and attributes valid for different Decision Data Models can be built. In this way, these components are re-usable for different decision models (e.g. in a hierarchy, an entity can be used in several decision model, or in different hierarchies, this entity can be used with other extended enterprise configuration using the same or new attributes).

Concerning the relationships between components in the decision modeling, these exist between the hierarchies defined with its levels (each hierarchy can have a different 


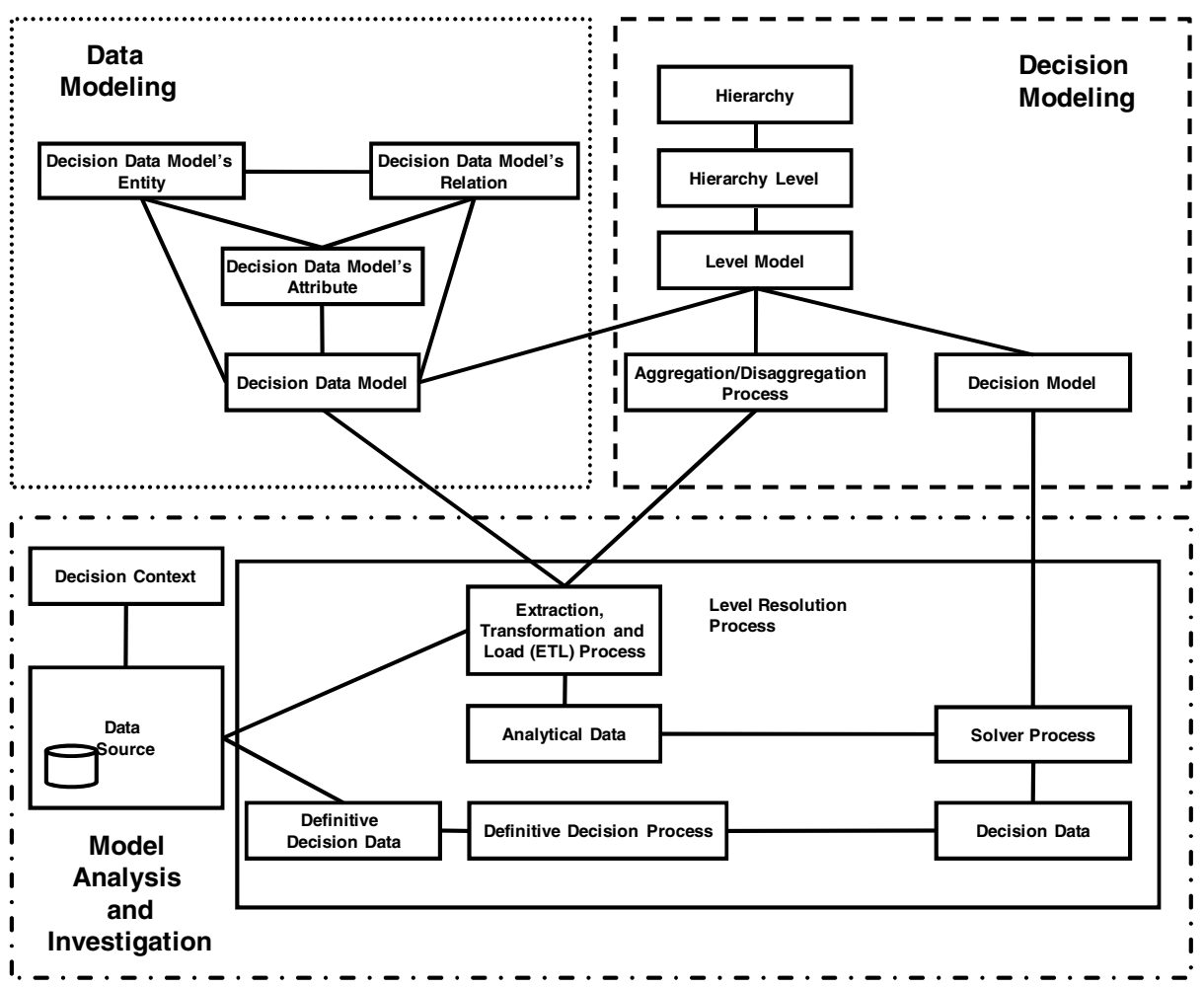

Fig. 1. Relationship between the components of the framework

number of levels). Also, each hierarchy level has associated a decision sub-problem in the level model. This level model encloses the Decision Data Model and the Decision Model necessary to solve a decision sub-problem. Moreover, aggregation/ disaggregation processes are associated with a Level Model to link information between lower and upper levels in a Hierarchy.

In this case also, it is possible to create libraries of Decision Models valid for different Hierarchies.

Finally, concerning the relationships between components in the Model Analysis and Investigation, the Level Resolution Process is used to solve a decision subproblem in an extended enterprise Decision Context. The Data Source in an extended enterprise decision context must be identified in order to Extract, Transform and Load all data necessary to instance the Decision Data Model. Analytical Data are obtained from this instantiation and completed with data obtained from the aggregation/disaggregation processes. The Solver Process use these Analytical Data for the resolution of a Decision Model and Decision Data are obtained. The decision-maker considers this information and additional factors in order to make a definitive decision (Definitive Decision Process). This Definitive Decision Data can be included in the Data Sources of participating enterprises. 


\subsection{Roles}

We have defined the main roles needed to model and operate this DSS. The roles identified are:

Table 1. Roles and Tasks

\begin{tabular}{|c|l|}
\hline Role & \multicolumn{1}{|c|}{ Task } \\
\hline Decision-Maker & $\begin{array}{l}\text { Person(s) in charge of a decision making for a } \\
\text { certain level in a decision problem hierarchy. }\end{array}$ \\
\hline Decision Model Designer & $\begin{array}{l}\text { Person(s) in charge of constructing the suitable } \\
\text { Decision Models for each level. }\end{array}$ \\
\hline Information System Designer & $\begin{array}{l}\text { Person(s) in charge of constructing the suitable } \\
\text { Information System to give service to the } \\
\text { information necessities }\end{array}$ \\
\hline
\end{tabular}

In an extended enterprise context is necessary to assign these roles between the participating enterprises. The tasks assigned to each role will be detailed in the next section.

\subsection{DSS Platform}

To perform a HEEDM supported by a DSS is necessary to develop a software tool. This tool can be developed to be used in different HEEDM contexts. Once the tool will be built, a parameterization and customization to a particular HEEDM context is required. The defined roles must participate in this parameterization and customization process to configure, in an easy way, the DSS platform.

The extended enterprise have usually at the heart of its organization a large final assembly plant or a service company procured by its suppliers $\left(1^{\text {st }}\right.$ tier supplier, $2^{\text {nd }}$ tier supplier) and serviced by its engineering units, sales units, banks, etc. [4], this main organization must encourage to the other extended enterprise participating to built and use this DSS Platform.

\section{Built a DSS Platform}

This platform must include the Data Model, Decision Model and Model Analysis and Investigation constructors of the hierarchical models (Fig. 2), that is, this platform must allow to include information about the structural components in a hierarchy. Also, the Aggregation/ Disaggregation Process, Extract, the Transformation and Load (ETL) Process, Solver Process or Definitive Decision Process can be included in this platform or can be outside but linked to this platform. To locate the process outside the platform can increase the flexibility if it is possible to change or adapt this process in an easier way.

A variety of software visions with interoperability orientation can be used in its implementation: Plug and Play Business Software [31], an integrated, unificated or federated structure [32], the implementation of the interoperability framework [33], the design of Decision Support System for Extended Enterprise [5], the information repository [34] or the Enterprise Model Management [35]. 


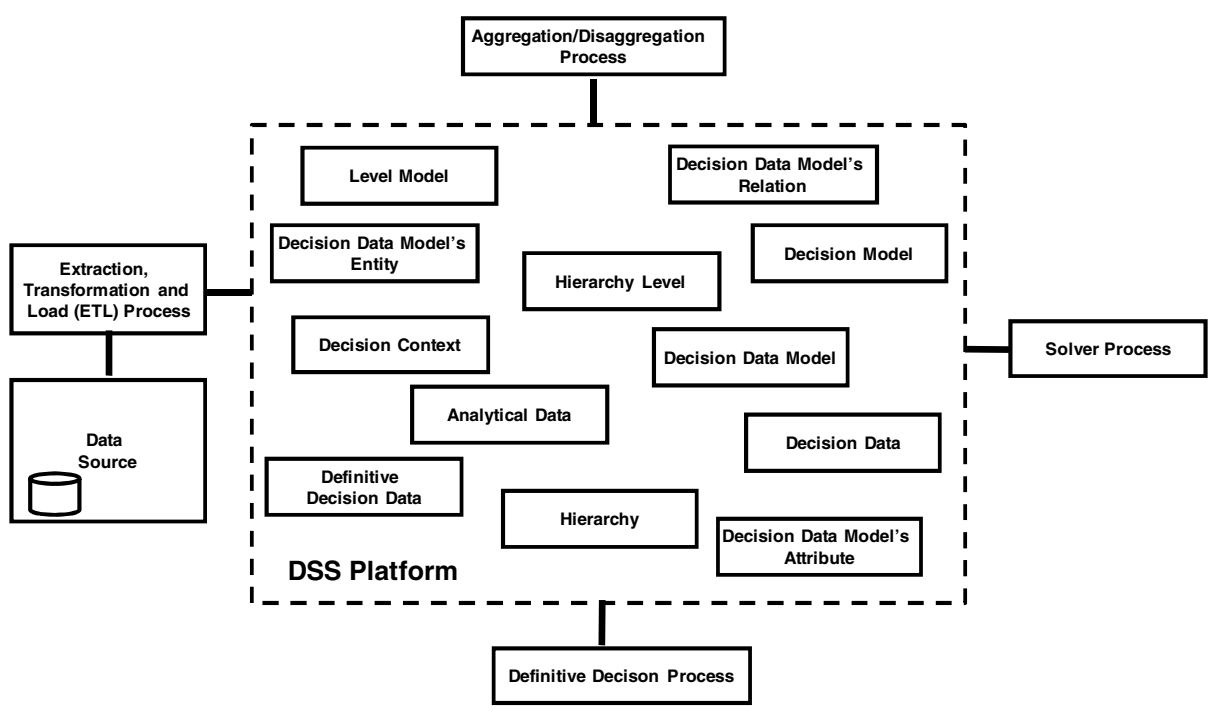

Fig. 2. DSS Platform

\section{Use of the DSS Platform for Modeling and Operations}

Modeling. Decision makers must use the platform to include information about a new hierarchy. Information about the Decision Data Model and the Decision Model for this hierarchy must be incorporated in the platform.

Decision Model Designer helps the Decision Maker in order to make the suitable Decision Models for each level in the hierarchy, the aggregation/disaggregation process and decide the solver process to use.

Information System Designer helps the Decision Maker to connect the DSS Platform with the Data Sources with the Extraction, Transformation and Load (ETL) Process and connect the DSS Platform with the other process (Aggregation/Disaggregation Process, Solver Process and Definitive Decision Process).

Operations. Only the Decision Maker in each level uses the DSS Platform to obtain results and decide yours definitive decision. The components included in the model analysis and investigation are used to operate with them to obtain Definitive Decision Data. The DSS Platform must manage these components and launch the necessary processes.

Remodeling. A new goal, changes in the current objectives, or small changes in the extended enterprise configuration, produce a necessary adjustment in the DSS. Depending on the type of change will be necessary only the decision maker participation, or Decision Model Designer and/or Information System Designer for more complex changes.

Simulations. Simulation scenarios can be used by the decision makers when they define fictitious hierarchies where they can play with real (or fictitious) organizational data and use different decision models. 


\section{Interoperability Aspects}

The proposed framework for a Decision Support System in a hierarchical extended enterprise decision context impacts into some interoperability aspects: enterprise levels, interoperability barriers and maturity models.

Although an extended enterprise context must have some interoperability characteristics solved, we approached these aspects from a general point of view.

The interoperability is achieved if the interaction between two enterprise systems can take place at various enterprise levels: data, services, processes and business [4]. The proposed framework includes: 1) the interoperability of data, in order to find and sharing information from heterogeneous data sources. 2) the interoperability of services, composing and making various application functions together. 3) the interoperability of processes which is limited to decision processes, and not other kind of processes; and 4) the interoperability of business where is necessary to harmonize the inter-enterprise decision making process in a hierarchical mode.

About the interoperability barriers (conceptual, organizational and technological) [36] (Fig. 3). Conceptual barriers related to the problems of syntactic and semantic of

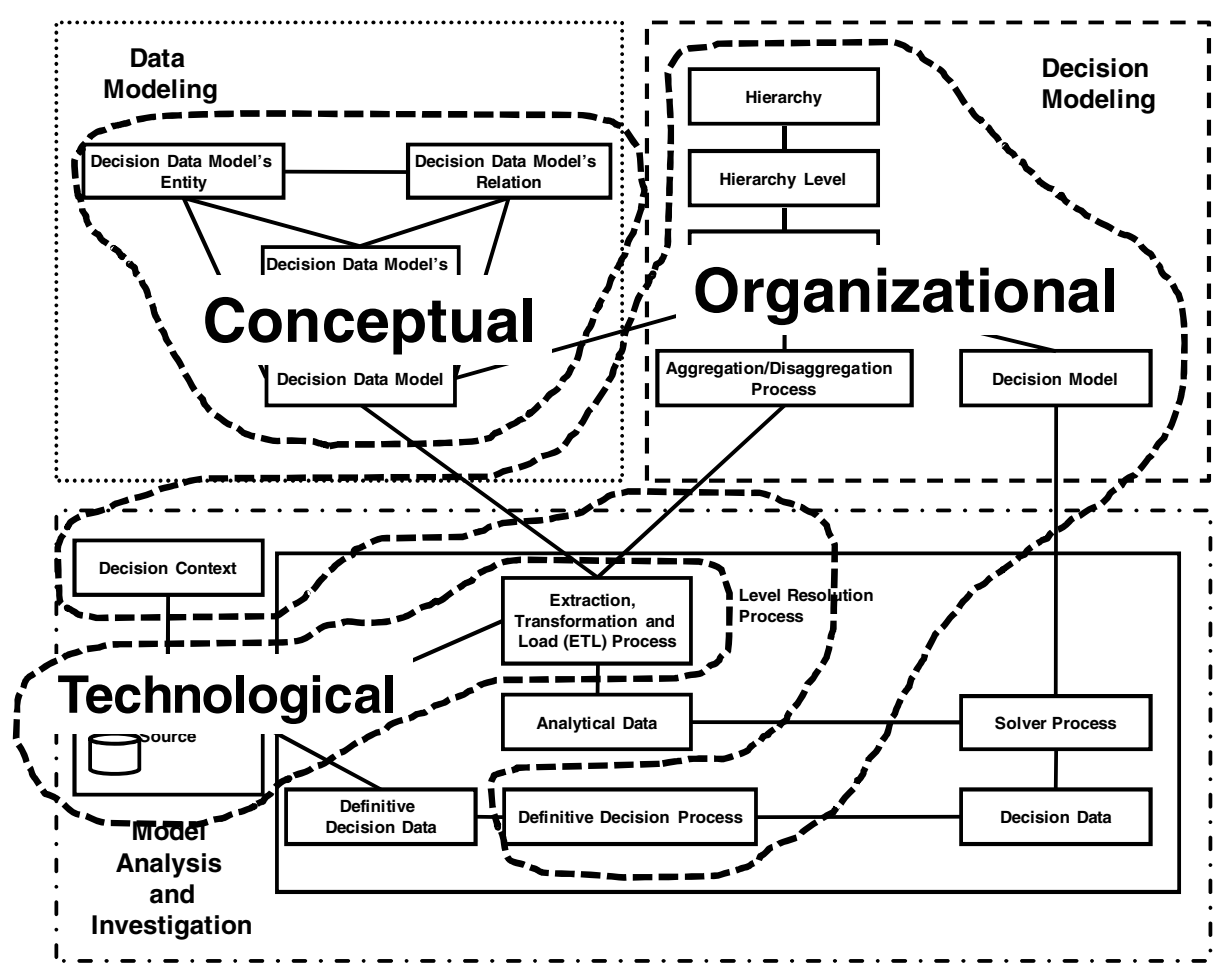

Fig. 3. Interoperability barriers 
information to be exchanged appears in the Data Modeling. Organizational barriers related to the definition of responsibilities and authority so that interoperability can take place under good conditions appears in the Decision Modeling, specifically in the hierarchy definition, levels, decision models and aggregation/disaggregation processes, also appears in the Decision Context as a particular setting which is necessary to identify and, finally appears in the Definitive Decision Process in order to identify the decision maker who make the definitive decision. And Technological barriers appears mainly in the Extraction, Transformation and Load Processes where the decision context include one, two or $\mathrm{n}$ data sources. This framework allows to identify and organize components to overcoming interoperability barriers and reuses these components for futures configurations.

With reference to the degree of integration of the considered enterprises, in [37] are identified some maturity models such as LISI, LCIM, OIM or EIF. In order to carry out a HEEDM supported by a DSS, it is necessary to reach the necessary integration level of the maturity models for each shared decision process.

\section{Conclusions}

The proposed framework allows identifying components for a DSS in an interenterprise decision context. The framework structure (Data modeling, Decision modeling, and Model analysis and investigation) has allowed organizing the identified components according to its characteristics.

The close relationships between participating enterprises in an extended enterprise are a key factor in this framework. These relationships are for a long term integration and more stable than other inter-organizational configurations. In this context is possible to extent the Hierarchical Production Planning structure to a Hierarchical Extended Enterprise Decision Making. A hierarchical inter-organizational decision making is not possible between organization without conceptual, organizational and technological links. The more stable context in extended enterprise allows build a DSS with the necessary conceptual, organizational and technological links for a long term hierarchical decision making, but flexible and agile to adapt to environment changes.

The framework allows working in the design process of a DSS in a HEEDM context. In this sense, it is possible use this framework in order to made a flexible DSS that can be used in a variety of settings in where a hierarchical approach allows an improvement in the decision making. The identification of these components is essential for its reusability. A flexible DSS can reuse components for futures configurations. In order to obtain a comprehensible framework with components easily identifiable, we have not include other factors like the user inferface (data presentations and interaction processes), the consistency problems associated with the aggregation/disaggregation process, the interval of time after which the set of decisions is reconsidered, or database structures (data warehouse, data marts, OLAP and so on).

A DSS in an extended enterprise decision context can increase the flexibility of the decision making in these enterprises where global regulatory and competitive factors rapidly change. Using this framework, a DSS can be designed to be parameterized and customized in an easy way for different context with reusable decision models and data access. 
An extended enterprise decision process implies communication and transactions between the participate enterprises, this paper review some interoperability aspects which impact in the proposed framework. In an HEEDM the interoperability barriers need to be overcome and this framework helps in this objective.

\section{References}

1. Shim, J.P., Warkentin, M., Courtney, J.F., Power, D.J., Sharda, R., Carlsson, C.: Past, present, and future of decision support technology. Decision Support System 33, 111-126 (2002)

2. Chen, D., Doumeingts, G.: European initiatives to develop interoperability of enterprise applications - basic concepts, framework and roadmap. Annual Reviews in Control 27, 153-162 (2003)

3. Jagdev, H.S., Browne, J.: The extended enterprise - a context for manufacturing. Production Planning \& Control 9(3), 219-229 (1998)

4. Chen, D., Doumeingts, G., Vernadat, F.: Architectures for enterprise integration and interoperability: Past, present and future. Computers in Industry 59, 647-659 (2008)

5. Zolghadri, M., Lecompte, T., Bourrieres, J.P.: Design of Decision Support Systems for Extended Enterprise. Studies In Informatics And Control With Emphasis on Useful Applications of Advanced Technology 11 (2002)

6. Hax, A., Meal, H.C.: Hierarchical Integration of Production Planning and Scheduling. Sloan Working Papers, pp. 656-673. ed. MIT, Cambridge (1973)

7. Bitran, G.R., Hax, A.C.: On the design of hierarchical production planning systems. Decision Science 8, 28-55 (1977)

8. Meal, H.C.: Putting Production Decisions Where They Belong. Harvard Business Review, Boston 62(2), 102-112 (1984)

9. Bitran, G.R., Tirupati, D.: Hierarchical Production Plannig. In: Graves, S.C., et al. (eds.) Handbooks in OR\&MS, vol. 4. Elsevier Science Publishers B.V., Amsterdam (1993)

10. McKay, K.N., Safayeni, F.R., Buzacott, J.A.: A Review of Hierarchical Production Planning and its Applicability for Modern Manufacturing. Production Planning \& Control 6, 384-394 (1995)

11. Vollmann, T.E., Berry, W.L., Whybark, D.C.: Sistemas de Planificación y Control de la Fabricación. IRWIN, Madrid (1995)

12. Schneeweiss, C.: Hierarchical Planning in Organizations: Elements of a general theory. International Journal of Production Economics 56, 547-556 (1998)

13. Vicens, E., Alemany, M.E., Andrés, C., Guarch, J.J.: A design and application methodology for hierarchical production planning decision support systems in an enterprise integration context. International Journal of Production Economics 74, 5-20 (2001)

14. Yan, H.: An interaction/prediction approach to hierarchical production planning and control with delay interaction. Computer Integrated Manufacturing Systems 10(4), 309-320 (1997)

15. De Kok, A.G., Fransoo, J.C.: Planning supply chain operations: Definition and comparison of planning concepts. In: Handbook in Operations Research and Management Science. Design and Analysis of Supply Chains, vol. 11. North-Holland, Amsterdam (2003)

16. Hurtubise, S., Olivier, C., Gharbi, A.: Planning tools for managing the supply chain. Computers \& Industrial Engineering 46, 763-779 (2004)

17. Alix, T., Zolghadri, M., Bourrieres, J.: A decision support system for production and procurement planning of enterprises X-networks. In: IEEE International Conference on Systems, Man and Cybernetics, vol. 5, pp. 4371-4376 (2004) 
18. Doumeingts, G., Pun, L., Mondain, M., Breuil, D.: Decision-making systems for production control planning and scheduling. International Journal of Production Research 16(2), 137-152 (1978)

19. Lario, F.C., Vicens, E.: Integrated System of MRP II Matriz-Based Hierarchical Planning: Its Computerized Implantation. In: Databases for Production Management. Elsevier Science Publishers, North-Holland (1990)

20. Davis, W.J., Thompson, S.D.: Production Planning and Control Hierarchy using a Generic Controller. IIE Transactions 25(4), 26-44 (1993)

21. Tsubone, H., Matsuura, H., Kimura, K.: Decision Support System for production planning -Concept and prototype. Decision Support Systems 13, 207-217 (1995)

22. Artiba, A., Aghezzaf, E.H.: An architecture of a multi-model system for planning and scheduling. International Journal of Computer Integrated Manufacturing 10(5), 380-393 (1997)

23. Wezel, W., Jorna, R.J.: The SEC-system reuse: support for scheduling system development. Decision Support Systems 26, 67-87 (1999)

24. Alemany, M.E.: Metodología y Modelos para el Diseño y Operación de los Sistemas de Planificación Jerárquica de la Producción. Aplicación a una Empresa del Sector Cerámico, $\mathrm{PhD}$, Universidad Politécnica de Valencia (2003)

25. Boza, A.: Propuesta de un Sistema de Información de Ayuda para la Toma de Decisiones en Planificación Jerárquica de la Producción, PhD, Universidad Politécnica de Valencia (2006)

26. Geoffrion, A.M., Maturana, S.: Generating optimization-based decision support systems. In: 28th Hawaii International Conference on System Sciences, vol. 3, pp. 439-448 (1995)

27. Makowski, M.: A Structured Modeling Technology. EJOR, Feature Issue on Advances in Complex System Modeling 166(3), 615-648 (2005)

28. Dominguez-Ballesteros, B., Mitra, G., Lucas, C., Koutsoukis, N.-S.: Modelling and solving environments of mathematical programming (MP): a status review and new directions. Journal of the Operation Research Society 53, 1072-1092 (2002)

29. Mayer, R.: IDEF1 Information Modeling. Knowledge Based Systems, Inc., College Station, Texas (1992)

30. Chen, P.: The entity-relationship model-toward a unified view of data. ACM Transactions on Database Systems 1(1), 9-36 (1976)

31. Jacobson, A., Davidsson, P.: An Analysis of Plug and Play Business Software. Department of Systems and Software Engineering, Blekinge Institute of Technology (BTH) Ronneby, Sweden. Springer, Boston (2007)

32. ISO 14258. Industrial automation systems - concepts and rules for enterprise models, TC 184/SC5/WG1, Geneva, Switzerland (1999)

33. Campos, C., Martí, I., Grangel, R., Mascherpa, A., Chalmeta, R.: A Methodological Proposal for the Development. In: Proceedings of MDISIS, pp. 47-57 (2008)

34. Sheth, A., Kalinichenko, L.: Information Modeling in Multidatabase Systems: Beyond Data Modeling of an Interoperability Framework. In: Finin, T.W., Yesha, Y., Nicholas, C. (eds.) CIKM 1992. LNCS, vol. 752. Springer, Heidelberg (1993)

35. Goul, M., Corral, K.: Enterprise model management and next generation decision support. Decision Support Systems 43, 915-932 (2007)

36. Daclin, N., Chen, D., Vallespir, B.: Methodology for Enterprise Interoperability. In: Proceedings of the 17th World Congress The International Federation of Automatic Control, Seoul, Korea (2008)

37. Panetto, H.: Towards a Classification Framework for Interoperability of Enterprise Applications. International Journal of CIM 20(8), 727-740 (2007) 